\title{
Analog-to-digital conversion beyond 20 bits
}

\author{
Applications, architectures, state of the art, limitations, and future prospects
}

\author{
Nikolai Beev \\ TE Department, EPC Group, HPM Section \\ CERN - European Organization for Nuclear Research \\ CH-1211 Geneva 23, Switzerland \\ E-mail: nikolai.beev@cern.ch
}

\begin{abstract}
Analog-to-digital converters with resolution exceeding 20 bits are widely available today. They exist in two forms - flexible laboratory instruments and monolithic integrated circuits. We present an overview of application fields for such digitizers, the hardware architectures that implement them, and their limitations. We review the state of the art by comparing the best-performing devices reported in scientific literature, as well as those available commercially. Our focus is particularly on integrated circuit solutions, and among our aims are the disambiguation of the multiple performance metrics in use, as well as the presentation of a clear perspective on basic performance tradeoffs. Finally, we provide a discussion on the future prospects for highresolution digitizing measurement systems.
\end{abstract}

Keywords-analog-to-digital, ADC, high-resolution, 20-bit, $L H C, H L-L H C$

\section{INTRODUCTION}

Modern electronics is predominantly digital. The progress in the development of microprocessors, digital memory, and programmable logic is best exemplified by Moore's law [1]. First stated in 1965, the law predicts exponentially increasing complexity and remains valid to this day. In the meantime, analog electronic devices have seen an altogether different path of development. In many aspects, they have reached physical and technical limitations that are difficult to overcome by conventional means. In precision mixed-signal circuits, the optimization of circuit area or power dissipation usually dictates a shift of complexity from the analog to the digital domain [2]. However, this approach has its limits too. Getting the best of both worlds requires a good understanding of the interfaces between them and the limitations they impose on the flow of information.

Non-electrical quantities of natural or artificial origin can have extremely high intrinsic dynamic range. In many cases, the first bottleneck in a measurement chain occurs due to the sensor or transducer. The electrical quantities at the output of a sensor are subject to the same constraints faced by the subsequent analog processing stages: the inevitable presence of noise and range limits imposed by device physics, technology, or convention. In certain cases, the sensor presents a challenge for the subsequent chain - for instance in photometry [3], magnetometry [4], or seismic instrumentation [5]. All practical analog signal conditioning stages (amplifiers, filters, etc.) are yet another information channel with limited capacity. Dynamic range bottlenecks are frequently avoided on the system level by methods such as sub-ranging, compensation, fine-coarse measurement, or nonlinear conversion. Yet, there are applications where such solutions are not optimal - for instance, when dealing with dynamic signals of considerable bandwidth, or where high linearity and stability are required throughout the full measurement range.

Analog-to-digital converters (ADCs) are the final, and sometimes ultimate bottleneck in the signal chain. One of the major trade-offs in digitization is between speed and resolution. It is due to a number of technical and quasi-fundamental limitations in the analog domain, such as noise, clock jitter, and supply voltages [6]. Several ADC architectures, as well as their multiple variations, have been developed and optimized to address specific application requirements. The scope of this work is on devices having nominal resolution beyond 20 bits, or dynamic range in excess of $120 \mathrm{~dB}$. Such ADCs are normally used to sample signals with a limited bandwidth in the range of $\mathrm{kHz}$ or below. The dominant performance limitation in these converters is excess noise, often modelled as an equivalent input-referred generator of thermal noise [6]. High-resolution ADCs exist in different forms, but in this work we are mostly concerned with commercial monolithic integrated circuits (ICs).

\section{APPLICATIONS}

A brief summary of the application fields for high-resolution ADCs, together with their specific requirements, is given in Table I. One common feature, apart from the obvious need for high dynamic range, is the relatively low importance of power dissipation in such devices. This fact is in marked contrast with the scientific aspect of ADC design, where typically a composite figure-of-merit that includes power dissipation is optimized on the system level [6].

\section{TABLE I. APPLICATION FIELDS OF HIGH-RESOLUTION DIGITIZERS}

\begin{tabular}{|l|l|l|l|}
\hline $\begin{array}{c}\text { Application } \\
\text { field }\end{array}$ & $\begin{array}{c}\text { Signal } \\
\text { bandwidth }\end{array}$ & Main requirements & Examples \\
\hline Audio & $\begin{array}{l}20 \mathrm{~Hz}-20 \\
\mathrm{kHz}\end{array}$ & $\begin{array}{l}\text { low distortion } \\
\text { low noise }\end{array}$ & {$[7]$} \\
\hline $\begin{array}{l}\text { Seismology / } \\
\text { geophysical } \\
\text { surveying }\end{array}$ & $\begin{array}{l}\text { near-DC to } \\
10^{2}-10^{3} \mathrm{~Hz}\end{array}$ & low distortion & {$[5]$} \\
\hline
\end{tabular}

Research supported by the High Luminosity LHC project. 


\begin{tabular}{|l|l|l|l|}
\hline $\begin{array}{l}\text { Industrial } \\
\text { instrumentation }\end{array}$ & near-DC & $\begin{array}{l}\text { EMI robustness } \\
\text { resolution } \\
\text { provisions for signal } \\
\text { conditioning } \\
\text { stability }\end{array}$ & {$[7][8]$} \\
\hline $\begin{array}{l}\text { Voltage } \\
\text { metrology }\end{array}$ & DC & $\begin{array}{l}\text { voltage range }> \pm 10 \mathrm{~V} \\
\text { linearity } \\
\text { stability }\end{array}$ & {$[9][10]$} \\
\hline $\begin{array}{l}\text { Electrical } \\
\text { power } \\
\text { metrology }\end{array}$ & $10^{4} \mathrm{~Hz}$ & $\begin{array}{l}\text { linearity } \\
\text { stability }\end{array}$ & {$[9][11]$} \\
\hline $\begin{array}{l}\text { High-precision } \\
\text { current control }\end{array}$ & near-DC & voltage range $\geq \pm 10 \mathrm{~V}$ & {$[13][14]$} \\
& & $\begin{array}{l}\text { resolution } \\
\text { linearity }\end{array}$ & \\
& & stability & \\
\hline
\end{tabular}

Audio ADCs are exclusively of the $\Sigma \Delta$ type and are typically not useful for other applications due to their very poor DC characteristics [7].

The instruments used in seismology and geophysical surveying require high dynamic range and linearity, but less bandwidth than audio. The DC performance is not of prime importance, although the low frequencies near DC may be of interest. Some unique requirements may apply, for example an extreme temperature range. Presently, there are seismic sensors with such high dynamic range, that even a very high-resolution ADC presents a bottleneck in the acquisition system [5].

In industrial sensor instrumentation, the suppression of EMI is one of the main objectives. Therefore, standard and wellproven techniques such as bridge circuits, ratiometric measurement, 4-wire connections and balanced/differential signaling are commonplace [7]. The manufacturers of modern integrated ADCs for industrial applications offer such features with the aim to ease the overall system design. Many ADCs have built-in analog multiplexers, programmable gain amplifiers (PGAs), 50/60 Hz notch filters and self-calibration capabilities. Digital signal processing techniques are common as well, thanks to the high availability and configurability of digital resources. Typical sensors that require high-resolution digitization include pressure and force gauges, weight cells, resistive thermometers, and thermocouples.

The digitizing devices suitable for metrology are primarily designed for stability, i.e. low variation of parameters with time and environmental factors. The static errors are less important, as they are subject to calibration. DC voltage metrology requires devices that can be used with $10 \mathrm{~V}$-output transfer standards and calibrators. One important advantage of having such wide voltage range is the reduced effect of thermal EMFs generated inadvertently at the contact points of dissimilar metals due to temperature gradients (Seebeck effect). This effect presents one of the most challenging limitations of DC and near-DC voltage measurements.

The scientific applications of high-resolution ADCs are very diverse. They include the readout of sensors [5] [8] [15], intrinsically low-noise devices like superconducting interferometers (SQUIDs) [16], particle accelerator beam instrumentation [17], digital integrators for pickup coil-based magnetic measurements [18] and quench detection in superconducting magnets [19]. Such applications are often driven by highly specific requirements that cannot be summarized.

High-precision current control for accelerator magnets [14], as a sub-field of scientific applications, is particularly demanding. The simultaneous requirements for stability, linearity, and resolution are high, since they all determine the overall control loop performance. The response to dynamic signals is important, but the requirements are relaxed in comparison with the static set point regime. Dynamic limitations exist due to other parts of the control loop - the load (magnet) and the actuator (power converter). Operation within a closed feedback loop also sets a limit on the measurement latency and the available time for signal processing.

The work we present is triggered by the needs of the High Luminosity LHC project [20] at CERN.

\section{HIGH-RESOLUTION ADC ARCHITECTURES}

There are presently three common architectures that implement high-resolution ADCs: (A.) multi-slope integrating, (B.) Sigma-Delta, and (C.) Successive Approximation Register (SAR). Their basic topologies are shown in Fig. 1.

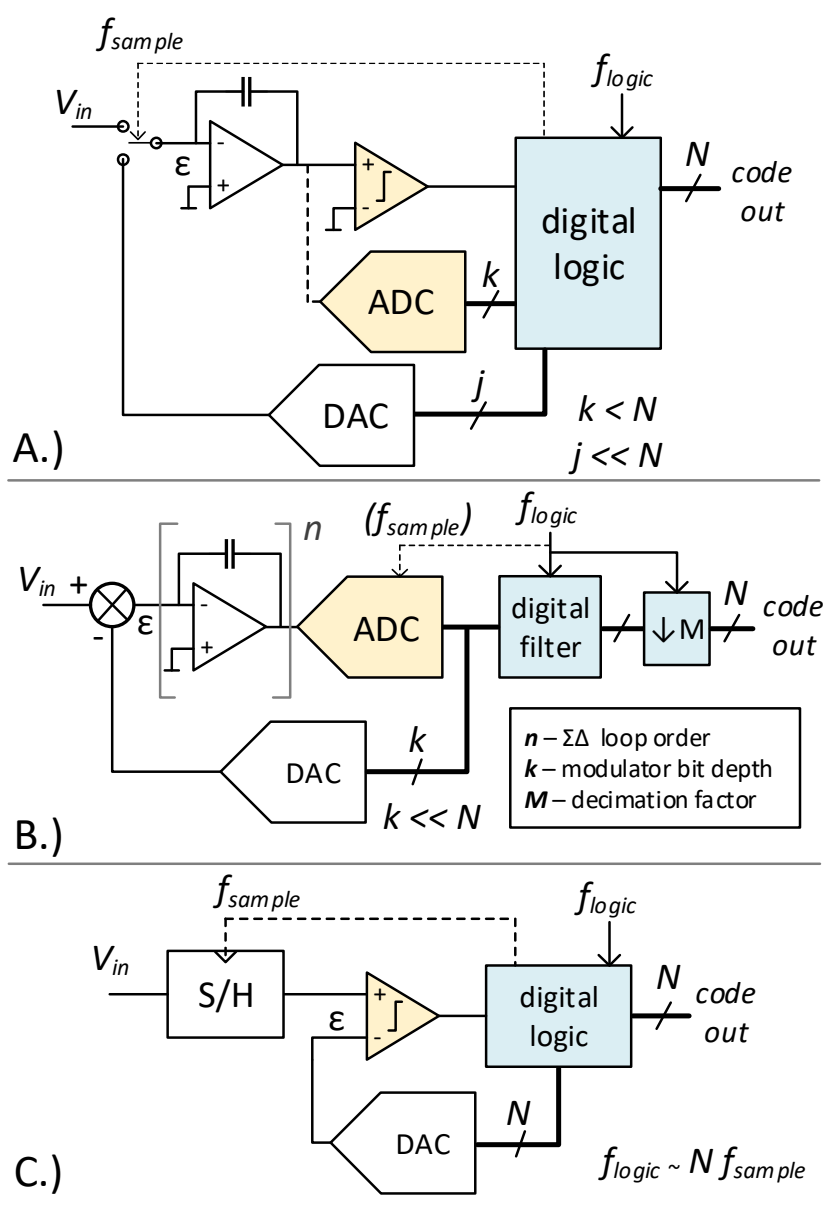

Fig. 1. Architectures implementing high-resolution ADCs: A.) multi-slope integrating; B.) Sigma-Delta; C.) SAR 
Conceptually, the three architectures are very similar - they are all based on a closed-loop circuit topology with a DAC in the feedback path and at least one low-resolution ADC (or comparator) in the loop. Architectures (A.) and (B.) also share similarities in the way they process information - both of them use the analog input signal to modulate a quantity that is later recovered by digital means. In contrast, SAR is simply a way to "invert" a DAC by incorporation into a feedback loop. As such, it has the least complex topology and most intuitive operation. Another common feature is that all three architectures have their digital logic operating at frequencies well above the sampling rate or output data rate (ODR). Their differences are summarized in Table II.

TABLE II. COMPARISON OF THE THREE HIGH-RESOLUTION ADC ARCHITECTURES

\begin{tabular}{|c|c|c|c|}
\hline & $\begin{array}{l}\text { Multi-slope } \\
\text { integrating }\end{array}$ & Sigma-Delta & SAR \\
\hline $\begin{array}{l}\text { In-loop DAC } \\
\text { resolution }\end{array}$ & $>1$ bit & $\geq 1$ bit & $\begin{array}{l}\text { N bits } \\
\text { (nominal res.) }\end{array}$ \\
\hline Error signal & $\varepsilon \rightarrow 0$ at $\mathrm{EOC}^{\mathrm{a}}$ & $\varepsilon(t) d t \rightarrow 0$ & $\varepsilon< \pm 1 / 2$ LSB at EOC \\
\hline Speed & slowest & mid-range to fast & fastest \\
\hline Latency & single cycle & $\begin{array}{l}\text { determined by } \\
\text { digital filter }\end{array}$ & single cycle \\
\hline Unique features & $\begin{array}{l}\text { synchronization } \\
\text { to power line } \\
\text { frequency }\end{array}$ & $\begin{array}{l}\text { quantization } \\
\text { noise shaping }\end{array}$ & $\begin{array}{l}\text { easy to multiplex } \\
\text { and interleave }\end{array}$ \\
\hline IC realizations & no & yes & yes \\
\hline $\begin{array}{l}\text { Typical } \\
\text { representatives }\end{array}$ & $\begin{array}{l}\text { Keysight } \\
3458 \mathrm{~A} \\
\text { Fluke 8508A } \\
\text { Keithley 2182A }\end{array}$ & $\begin{array}{l}\text { AD7177-2 } \\
\text { ADS1281 } \\
\text { MAX11210 }\end{array}$ & $\begin{array}{l}\text { AD7767 } \\
\text { LTC1280-24 } \\
\text { MAX11905 }\end{array}$ \\
\hline
\end{tabular}

a. End of Conversion

Multi-slope integrating ADCs are known to engineers and scientists mainly in the form of high-grade tabletop digital voltmeters (DVMs), such as the 8.5-digit devices HP/Agilent/Keysight 3458A and Fluke 8508A. Presently, there are no monolithic IC realizations of this architecture. Lowerresolution dual-slope integrating ADCs do exist in IC form, but their performance does not approach the 20-bit level.

Most high-resolution ADCs today are of the Sigma-Delta type. They include devices published in scientific literature, commercial digitizer systems, and commercial ICs. There are many variations of the architecture [21], but the highestresolution devices are typically based on single- or multi-bit modulators of $3^{\text {rd }}$ or $4^{\text {th }}$ order operating at oversampling ratios of $\approx 10^{2}-10^{4}$. The highest effective resolution figures are achieved within narrow frequency bands, where the noise of the in-loop quantizer is suppressed to very low levels, and where other noise mechanisms dominate. A variation of the Sigma-Delta architecture, known as incremental $A D C$, has also been reported in scientific literature as a viable solution for high-resolution high-accuracy digitization [22]. However, to the best of our knowledge, there are no commercial devices based on it.

SAR ADCs are the big winner of CMOS technology scaling [23]. It is due to their simplicity and inherent scalability, as well as the fact that a typical SAR realization does not require any linear analog stages; all functional blocks can be implemented using switched capacitors and charge redistribution techniques. As a result, SAR devices cover a very broad range on the ADC landscape, either in standalone form, or as building blocks for other architectures (pipelined and time-interleaved converters). In the recent years, the SAR architecture has presented very competitive high-resolution ICs as well. However, to reach effective resolution figures beyond 20 bits, they must operate in oversampling mode. Some ICs have built-in provisions for it, while for others it has to be implemented in external logic.

\section{DEFINITIONS AND PERFORMANCE METRICS}

\section{A. Overview}

The lack of standardization in the field of high-resolution ADCs, as well as the diverging application goals, have resulted in the existence of multiple ways to define and quantify performance. In this section we review the commonly used metrics. We note that authors of scientific articles, books, and technical documentation often do not make clear distinction between the terms, or use them interchangeably.

The quantities given below refer either to the full-scale peak-

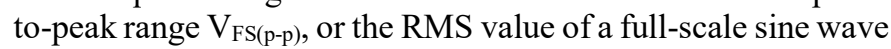

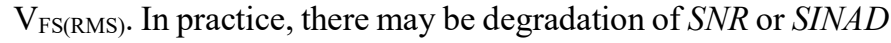
for signals that approach the range limits; at this point we ignore such effects and assume maximum $S N R$ at 0 dBFS.

- $\quad$ Signal-to-Quantization Noise Ratio $(S Q N R)$

$$
\operatorname{SQNR}[d B]=10 \log _{10} \frac{P_{\text {signal }}}{P_{\text {quant. noise }}}=6.02 N+1.76
$$

- $\quad$ Signal-to-Noise Ratio $(S N R)[6]$

$$
\operatorname{SNR}[d B]=10 \log _{10} \frac{P_{\text {signal }}}{P_{\text {quant. noise }}+P_{\text {excess noise }}}
$$

- $\quad$ Signal-to-Noise and Distortion ratio (SINAD) [24]

$$
\operatorname{SINAD}[d B]=10 \log _{10} \frac{P_{\text {signal }}}{P_{\text {noise }}+P_{\text {distortion }}}
$$

- $\quad$ Effective Resolution $(E R)$

$$
E R[\text { bits }]=\log _{2}\left(\frac{V_{F S(p-p)}}{V_{\text {noise }(R M S)}}\right)
$$

- $\quad$ Noise-Free Code Resolution (NFCR)

$$
\begin{aligned}
\text { NFCR }[\text { bits }] & =\log _{2}\left(\frac{V_{F S(p-p)}}{V_{\text {noise }(p-p)}}\right)=E R-2.7 \text { bits } \\
\text { * } & \text { valid for } 1000 \text { samples, assuming noise with Gaussian distribution }
\end{aligned}
$$

- $\quad S N R$ bits [6]

$S N R$ bits $[$ bits $]=\frac{S N R[d B]-1.76}{6.02}$

- $\quad$ Effective Number of Bits $(E N O B)[24]$

$E N O B[$ bits $]=\frac{\operatorname{SINAD}[d B]-1.76}{6.02}$ 


\section{B. Signal-to-noise ratios}

$S Q N R$ is the signal-to-noise ratio for a full-scale pure sine wave digitized by an ideal N-bit Nyquist-rate ADC. Any practical ADC has excess noise, which leads to the common SNR metric. SINAD is yet stricter, as it includes distortion products due to non-linearity of the digitizer. In the context of highresolution ADCs, it may be impractical or even impossible to measure directly SINAD due to the lack of sine wave generators with sufficiently low distortion [25]. For any practical ADC with $\mathrm{N}>20$ bits, excess noise dominates over quantization and determines $S N R$ at high data rates. Oversampling, decimation, and filtering provide a way to gain equivalent resolution that matches or exceeds the nominal.

\section{Equivalent Resolution}

The four given ways to specify equivalent resolution count on different assumptions. ER and NFCR do not assume a dynamic signal; they are simply a way to refer excess ADC noise to the full-scale range. As such, they have no directly corresponding $S N R$-like quantities, and are normally measured with shorted $\mathrm{ADC}$ inputs, i.e. a $0 \mathrm{~V}$ noiseless $\mathrm{DC}$ input voltage. In some cases, excess noise is dependent on the DC input level [9] [16]; this effect is not reflected in the basic effective resolution metrics, but may be given separately in the form of plots, tables, etc.

In contrast, $S N R$ bits and $E N O B$ assume a full-scale sine wave test signal. These metrics correspond directly to $S N R$ and SINAD; they are essentially a way to lump together the converter non-idealities into the "quantization noise" of an equivalent ideal Nyquist-rate ADC.

\section{Noise}

Sometimes manufacturers specify the ADC excess noise separately from other metrics. It can be given in different forms: input-referred RMS noise voltage, input-referred noise spectral density, output-referred noise relative to $1 \mathrm{LSB}$, or output noise referred to the full-scale range (e.g. ppm $_{\mathrm{rms}}$ ). The true noise of the ADC core should be distinguished from the input-referred noise floor of any preceding stage. In case the ADC has a builtin PGA, the relevant noise specification is given for unity gain setting, or bypassed PGA. Knowing the input-referred noise density allows one to calculate the integrated RMS noise within a given bandwidth, and thus estimate the noise-limited $S N R$ or equivalent resolution at different data rates. This approach is particularly useful when digital post-processing, e.g. custom filtering with decimation, is implemented in external logic. The RMS noise values given in datasheets can be converted to noise density using the equivalent noise bandwidth of the used digital filter, which is usually of the $\sin c^{n}$ type. For a given filter type, this bandwidth is a fixed fraction of the ODR.

\section{E. Static errors and drifts}

Many applications require not only high resolution, but also an accordingly good accuracy. It is quantified by a number of DC parameters. They can be subdivided into static errors, drifts, linear, and non-linear ADC transfer function parameters. The parameters are either referred to the input or the output, and they can be given in absolute or relative units. The drift terms are given in input-referred absolute or relative units per degree
Celsius. In some cases, the temperature dependence of nonlinearity is given, either as worst-case INL as a function of temperature, or in plot form of INL versus code, with temperature as a parameter.

\section{STATE of THE ART}

A summary of the best ADCs reported in scientific literature in terms of dynamic range is given in Table III. Table IV compares the absolute noise floor and metrology-relevant DC characteristics of a number of commercial high-resolution ADCs. Fig. 2 shows a plot of noise-limited SNR versus bandwidth both for commercial and scientific ADCs.

TABLE III. BEST-PERFORMING ADCS REPORTED IN SCIENTIFIC LITERATURE

\begin{tabular}{|l|l|l|l|}
\hline \multicolumn{1}{|c|}{ Publication } & \multicolumn{1}{|c|}{$\begin{array}{c}\text { Noise floor } \\
{[\mathbf{n V} / \sqrt{\mathbf{H z}}]}\end{array}$} & $\begin{array}{c}\text { Full-scale } \\
\text { range } \\
{\left[\mathbf{V}_{\mathbf{p}-\mathbf{p}}\right]}\end{array}$ & \multicolumn{1}{|c|}{ Note } \\
\hline Pett [13], [14] & 200 & 20 & $\Sigma \Delta, 3^{\text {rd } \text { order, single-bit }}$ \\
\hline Gao et al. [5] & 10 & 20 & $\begin{array}{l}\text { Multibit } \Sigma \Delta \text { built out of } \\
\text { a 16-bit DAC }\end{array}$ \\
\hline $\begin{array}{l}\text { Naiknaware, } \\
\text { Fiez [26] }\end{array}$ & 4 & 2.6 & $\begin{array}{l}\text { Integrated CMOS } \Sigma \Delta \\
\text { with CDS }\end{array}$ \\
\hline Qu et al. [27] & $9 \pm 2$ & 0.632 & $\begin{array}{l}\text { NI PXI-4462 }(\Sigma \Delta) \text { with } \\
\text { CDS }\end{array}$ \\
\hline
\end{tabular}

TABLE IV. NOISE, DRIFT AND NONLINEARITY OF SOME HIGHPERFORMANCE COMMERCIAL ADCS

\begin{tabular}{|l|l|l|l|l|l|l|}
\hline \multicolumn{1}{|c|}{ ADC } & Type & \multicolumn{1}{|c|}{$\begin{array}{l}\text { N } \\
{[\mathbf{b i t s}]}\end{array}$} & $\begin{array}{c}\text { Noise } \\
\mathbf{f l o o r} \\
{[\mathbf{n V} /} \\
\sqrt{\mathbf{H z}}]\end{array}$ & $\begin{array}{c}\text { Offset } \\
\mathbf{d r i f t} \\
{[\mathbf{p p b} /} \\
\left.{ }^{\circ} \mathbf{C}\right]\end{array}$ & $\begin{array}{c}\text { Gain } \\
\mathbf{d r i f t} \\
{[\mathbf{p p m} /} \\
\left.{ }^{\circ} \mathbf{C}\right]\end{array}$ & $\begin{array}{c}\text { INL } \\
\text { (typ) } \\
{[\mathbf{p p m}]}\end{array}$ \\
\hline AD7177-2 & SD & 32 & $30^{\mathrm{a}}$ & \pm 8 & \pm 0.4 & \pm 1 \\
\hline ADS1256 & SD & 24 & 120 & \pm 20 & \pm 0.8 & \pm 3 \\
\hline ADS1262 & SD & 32 & 110 & \pm 0.1 & 0.5 & 3 \\
\hline ADS1281 & SD & 32 & 110 & 6 & 0.4 & 0.6 \\
\hline AD7190 & SD & 24 & $280^{\text {a }}$ & \pm 0.5 & \pm 1 & \pm 5 \\
\hline LTC2440 & SD & 24 & 70 & \pm 2 & 0.2 & 5 \\
\hline CS5532 & SD & 24 & 70 & \pm 1 & \pm 2 & \pm 15 \\
\hline ISL26104 & SD & 24 & 120 & \pm 30 & \pm 0.1 & \pm 2 \\
\hline MAX11210 & SD & 24 & $250^{\mathrm{b}}$ & 5 & 0.05 & \pm 10 \\
\hline LTC2508-32 & SAR & 32 & 50 & \pm 14 & \pm 0.05 & \pm 0.5 \\
\hline LTC2380-24 & SAR & 24 & $30^{\mathrm{b}}$ & 9 & 0.05 & 0.5 \\
\hline AD7767 & SAR & 24 & 60 & 1.5 & 0.4 & 3 \\
\hline
\end{tabular}

\section{LIMITATIONS}

\section{A. Signal range}

Most high-resolution integrated ADCs have differential inputs. This feature allows for a full-scale range equal to twice the reference voltage. A de facto standard for external reference voltage of commercial high-resolution ADCs is $5 \mathrm{~V}$; therefore, most converters have a full-scale range of $10 \mathrm{~V}_{\mathrm{p}-\mathrm{p}}$. The devices 


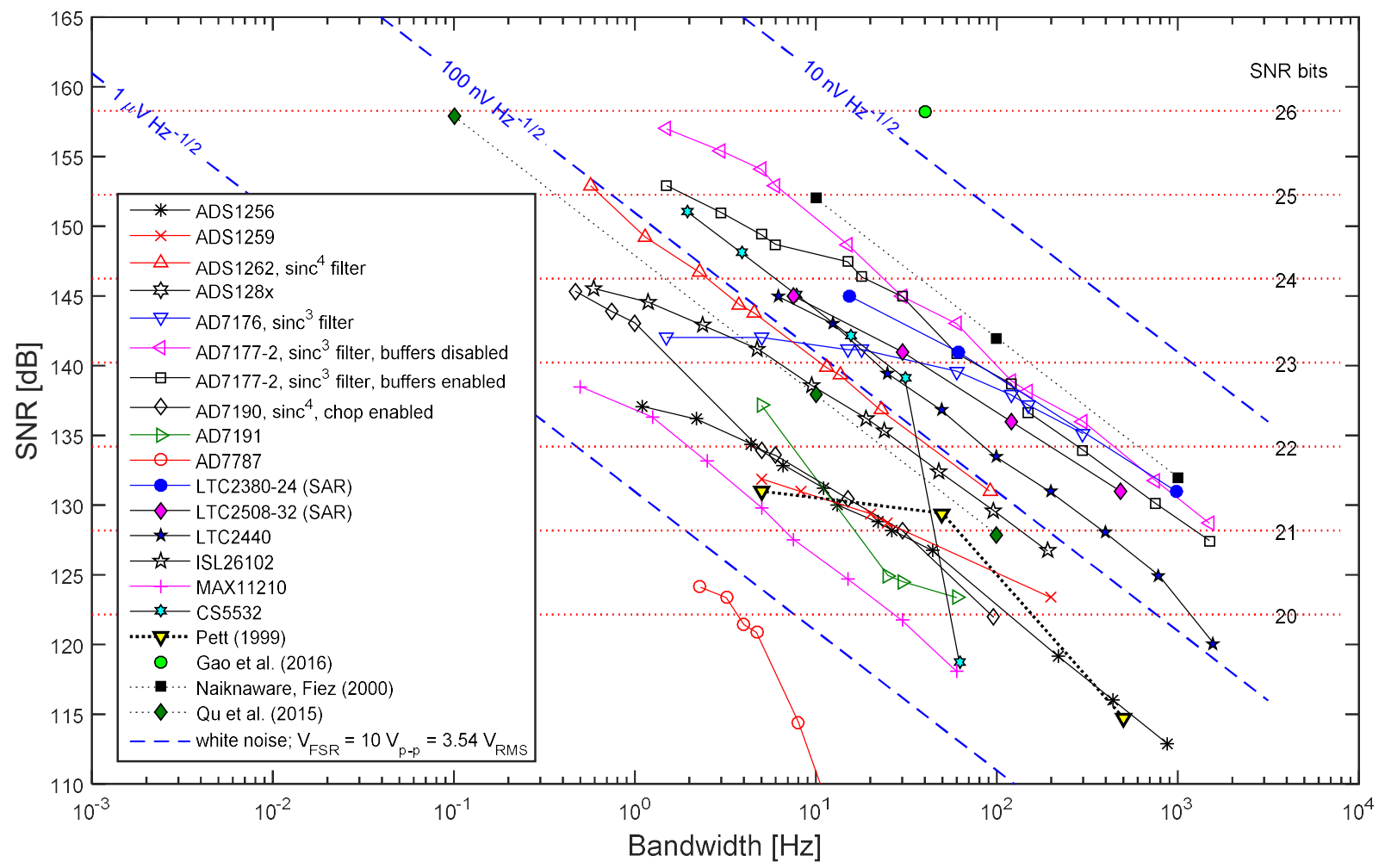

Fig. 2. Comparison of high-resolution scientific and commercial ADCs in terms of SNR and SNR-equivalent resolution (SNR bits)

reported in scientific literature usually do not follow the same pattern - they exhibit either application-specific [5], or technology-limited ranges [26]. When the range of the measured signal does not match the ADC input full scale, the analog input conditioning stages must either amplify or attenuate the signal. Special care must be taken to ensure that the scaling of the input signal and reference voltage does not degrade the system dynamic range, linearity, and other aspects of performance.

\section{B. Noise}

Broadband noise is the main resolution-limiting factor at data rates above a few Hz. This noise usually has frequencyindependent (white) spectral density, because it has fundamental origins (thermal or shot noise). It is relatively easy to reduce the broadband noise contribution of the reference voltage to negligible levels by low-pass filtering; the remaining figure is the intrinsic noise floor of an ADC core. For devices with a builtin PGA, the overall input-referred noise is minimized at moderately high PGA gain. However, in this regime the system dynamic range is degraded, due to the reduced full-scale input range.

Low-frequency (flicker, 1/f) noise is the main limiting factor for measurements at longer time scales, corresponding to narrowing the measurement bandwidth down to a few $\mathrm{Hz}$ or less. Fig. 2 shows that certain devices have a very low $1 /$ f noise corner frequency. This fact is either due to special features like built-in auto-zeroing or chopping stages, or is a merit of the circuit realization. Techniques like correlated double sampling (CDS) can be employed to effectively suppress $1 / \mathrm{f}$ noise [26], at the expense of extra circuitry and added latency [27].

\section{Reference voltage source}

Some integrated-circuit ADCs contain a built-in bandgap reference voltage source. Although they have seen much progress in recent years, their performance is not comparable to external buried-zener references in terms of noise, temperature dependence, and long-term drift. The unchallenged leader in this distinctly stagnated field is still the LTZ1000, with specified temperature coefficient of $0.05 \mathrm{ppm} /{ }^{\circ} \mathrm{C}$ and long-term stability better than $1 \mathrm{ppm} / \sqrt{k H r}[28]$.

\section{Drift and Linearity}

Many high-resolution integrated ADCs exhibit excellent temperature stability. The typical offset drift is in the range of $\mathrm{nV} /{ }^{\circ} \mathrm{C}$, which corresponds to part-per-billion (ppb) levels of the full scale. Gain drift is significantly worse, but for some devices it is well below $1 \mathrm{ppm} /{ }^{\circ} \mathrm{C}$, therefore not far from the drift of the best available reference voltage source. For ultimate performance, an external temperature-stabilizing loop may be employed [13].

The best specified INL levels are below $1 \mathrm{ppm}$. It is not realistic to expect significant decrease in this figure due to process improvement alone; advanced techniques like largescale dither [15] or multi-point calibration can improve linearity.

\section{DISCUSSION}

It is nowadays possible to build digitizers for demanding applications that are based on commercial integrated highresolution ADCs [8] [9] [11] [12] [16] [18]. However, reaching 
a level of accuracy comparable to the resolution beyond 20 bits remains a difficult task that requires careful consideration of many system-level factors. Furthermore, even though ADCs with nominal resolution of 24 bits are prolific, few parts can actually achieve $S N R$-equivalent resolution beyond 24 bits.

It is hard to predict the rate of improvement in resolution and accuracy for commercial high-resolution ADCs, as their segment is not subject to the strongest market pressure. The dominance of the Sigma-Delta architecture is evident, although a number of SAR ADCs are catching up. Customized and hybrid digitizers [5] [27] are still capable of achieving higher dynamic range than fully integrated solutions.

The most demanding high-resolution applications could benefit from the unique analog signal processing capabilities of some sensors and circuits. Piecewise linear or non-linear analog stages have been used for range compression in systems with low accuracy requirements [29]. Sensors with periodic output could perform dynamic range folding, effectively relieving the ADC bottleneck. This approach has been demonstrated with SQUIDs [4] [30], and has been proposed for magneto-optical current sensors based on the Faraday effect [31].

We would like to point out that in the case of dynamic signals, the above-mentioned methods are essentially ways to exchange bandwidth for dynamic range. As such, they complement a number of established and well-known information-processing techniques (modulation, oversampling, dithering) that are at the very heart of existing high-resolution ADC technologies. One common feature of such techniques is that they all rely on digital post-processing. An informationtheoretic approach to the problem of digitization could shed more light on the optimal use of analog and digital resources in high-performance signal acquisition systems.

\section{REFERENCES}

[1] R. R. Schaller, "Moore's Law: past, present and future," IEEE Spectrum, pp. 53-59, 1997.

[2] R. Sarpeshkar, "Analog versus digital: extrapolating from electronics to neurobiology.," Neural Computation, vol. 10, no. 7, pp. 1601-37, 1998.

[3] G. Eppeldauer and E. Hardis, "Fourteen-decade photocurrent measurements with large-area silicon photodiodes at room temperature," Applied Optics, vol. 30, no. 22, pp. 3091-3099, 1991.

[4] C. Ludwig, C. Kessler, A. J. Steinforc and W. Ludwig, "Versatile high performance digital SQUID electronics," IEEE Transactions on Applied Superconductivity, vol. 11, no. 1, pp. 1122-1125, 2001.

[5] SH. Gao, B. Xue, J. Li, Z. Lin, Y. Chen and XY. Zhu, "High-resolution data acquisition technique in broadband seismic observation systems," Science China Technological Sciences, vol. 59, no. 6, pp. 961-972, 2016.

[6] R. H. Walden, "Analog-to-digital converter survey and analysis," IEEE Journal on Selected Areas in Communications, vol. 17, no. 4, pp. 539-550, 1999.

[7] P. Horowitz and W. Hill, The Art of Electronics, 3rd Ed., Cambridge University Press, 2015.

[8] R. Ambrosetti, R. Matteoli and D. Ricci, "Note: A versatile, stable, highresolution readout system for RTD and thermistor sensors," Review of Scientific Intruments, vol. 83, p. 096101, 2012.

[9] F. Overney, A. Rüfenacht, J.-P. Braun, B. Jeanneret and P. S. Wright, "Characterization of metrological grade analog-to-digital converters using a programmable Josephson voltage standard," IEEE Transactions on Instrumentation and Measurement, vol. 60, no. 7, pp. 2172-2177, 2011.

[10] J. R. Pickering, D. Georgakopoulos, J. M. Williams and P. S. Wright, "Design of a quantum-based oversampling delta-sigma analogue-to-digital converter for metrology applications," IEE Proceedings - Science, Measurement and Technology, vol. 151, no. 5, p. 362-367, 2004

[11] R. Iuzzolino, L. Palafox, W. G. K. Ihlenfeld, E. Mohns and C. Brendel, "Design and characterization of a sampling system based on $\Sigma-\Delta$ analog-to-digital converters for electrical metrology," IEEE Transactions on Instrumentation and Measurement, vol. 58, no. 4, pp. 786-790, 2009.

[12] P. S. Wright and P. Clarkson, "Development of an Ethernet-enabled digitizer for on-site AC measurements," IEEE Transactions on Instrumentation and Measurement, vol. 60, no. 7, pp. 2229-2234, 2011.

[13] J. G. Pett, "A high accuracy 22 bit sigma-delta converter for digital regulation of super-conducting magnet currents," in Advanced A/D and D/A Conversion Techniques and their Applications, Glasgow, UK, 1999.

[14] M. C. Bastos, G. Fernqvist, G. Hudson, J. Pett, A. Cantone, F. Power, A. Saab, B. Halvarsson and J. R. Pickering, "High accuracy current measurement in the main power converters of the Large Hadron Collider: tutorial 53," IEEE Instrumentation \& Measurement Magazine, vol. 17, no. 1, pp. 66-73, 2014.

[15] J. Sanjuán, A. Lobo and J. Ramos-Castro, "Analog-to-digital converters nonlinear errors correction in thermal diagnostics for the laser interferometer space antenna mission," Review of Scientific Instruments, vol. 80, p. 114901, 2009.

[16] Y. J. Kim, B. Kunkler, C.-Y. Liu and G. Visser, "A high dynamic range data acquisition system for a solid-state electron dipole moment experiment," Review of Scientific Instruments, vol. 83, no. 1, p. 013502, 2012

[17] A. Boccardi, M. Gasior, O. R. Jones, K. K. Kasinski and R. J. Steinhagen, "The FPGA-based continuous FFT tune measurement system for the LHC and its test at the CERN SPS," in Proceedings of PAC07, Albuquerque, New Mexico, USA, 2007.

[18] A. M. Batrakov and A. V. Pavlenko, "Precise digital integration in wide time range: theory and realization," Journal of Instrumentation, vol. 12, no. 7, pp. 07012-07012, 2017.

[19] R. Denz, E. de Matteis, A. Siemko and J. Steckert, "Next generation of quench detection systems for the High-Luminosity upgrade of the LHC," IEEE Transactions on Applied Supercondictivity, vol. 27, no. 4, pp. 1-4, 2017.

[20] L. Rossi and O. Brüning, The High Luminosity Large Hadron Collider. A new machine for illuminating the mysteries of universe, ser. Advanced series on directions in high energy physics, World Scientific Publishing Co Pte Ltd, 2015.

[21] J. de la Rosa, "Sigma-Delta modulators: tutorial overview, design guide, and state-of-the-art survey," IEEE Transactions on Circuits and Systems, vol. 58, no. 1, pp. 1-21, 2011 .

[22] J. Márkus, J. Silva and G. C. Temes, "Theory and applications of incremental $\Delta \Sigma$ converters," IEEE Transactions on Circuits and Systems - I: Regular Papers, vol. 51, no. 4, pp. 678 - 690, 2004.

[23] B. Murmann, "The successive approximation register ADC: a versatile building block for ultra-low-power to ultra-high-speed applications," IEEE Communications Magazine, vol. 54, no. 4, pp. 78-83, 2016.

[24] IEEE Std 1241-2010 IEEE Standard for terminology and test nethods for analog-to-digital converters, IEEE, 2010.

[25] J. Williams and G. Hoover, "AN132: Fidelity testing for A->D converters proving purity," Linear Technology, 2011.

[26] R. Naiknaware and T. Fiez, "142 dB $\Sigma \Delta$ ADC with a $100 \mathrm{nV}$ LSB in a $3 \mathrm{~V}$ CMOS process," in Proceedings of the IEEE 2000 Custom Integrated Circuits Conference, 2000. CICC., Orlando, FL, 2000.

[27] S.-B. Qu, O. Robert, P. Lognonné and Z.-B. Y. S.-Q. Zhou, "Low frequency noise elimination technique for 24-bit $\Sigma-\Delta$ data acquisition systems," Review of Scientific Instruments, vol. 86, no. 3, p. 034708, 2015.

[28] P. J. Spreadbury, "The ultra-zener - a portable replacement for the Weston cell?," IEEE Transactions on Instrumentation and Measurement, vol. 40, no. 2, pp. 343-346, 1991.

[29] G. Mészáros, C. Li, I. Pobelov and T. Wandlowski, "Current measurements in a wide dynamic range - applications in electrochemical nanotechnology," Nanotechnology, vol. 18, no. 42, p. 424004, 2007.

[30] M. Kiviranta and N. Beev, "Four-quadrant flux quanta counting for wide-range SQUID amplifiers," Superconductor Science and Technology, vol. 27, no. 7, p. $075005,2014$.

[31] D. Gerber and J. Biela, "High-dynamic and high-precise optical current measurement system based on the Faraday effect," IEEE Transations on Plasma Science, vol. 43, no. 10, pp. 3550-3554, 2015. 